Original Paper http://ajol.info/index.php/ijbcs http://indexmedicus.afro.who.int

\title{
Biodiversité végétale et valeur d'usage en zone soudanienne de la Côte d'Ivoire
}

\author{
Djakalia OUATTARA ${ }^{1}$, Djaha KOUAME ${ }^{2}$, Marie-Solange TIEBRE ${ }^{1 *}$, \\ Yao Jean-Clovis, KOUADIO ${ }^{1}$ et Kouakou Edouard N'GUESSAN ${ }^{1}$ \\ ${ }^{1}$ Laboratoire de Botanique, Université Félix HOUPHOUËT-BOIGNY, \\ 22 BP 582 Abidjan 22, Côte d'Ivoire. \\ ${ }^{2}$ UFR Environnement, Université Jean Lorougnon GUEDE, BP 150 Daloa, Côte d'Ivoire. \\ "Auteur correspondant ; E-mail : tiebrems@hotmail.com ; Tel : 46415181
}

\section{RESUME}

La végétation du Nord de la Côte d'Ivoire est en majorité dominée par des savanes qui ont fait l'objet de peu d'études botaniques. La présente étude réalisée à Tengrela dans le Nord de la Côte d'Ivoire a pour but d'évaluer la diversité des formations végétales et leurs utilisations par les populations locales. La collecte des données a combiné la méthode de relevé de surface et celle dite itinérante. Des enquêtes ethnobotaniques ont été menées auprès des tradipraticiens pour appréhender l'importance culturelle des espèces. L'étude a permis de mettre en évidence 6 formations végétales que sont les forêts claires, les forêts galeries, les jachères, les savanes arborées, les mares et les savanes herbeuses. On dénombre 402 espèces réparties en 269 genres et 84 familles dont 22 espèces à statut particulier. Il ressort de l'enquête que plusieurs espèces sont utilisées par les populations locales pour la consommation, le traitement des maladies, et divers autres usages. Les espèces à usage alimentaire se récoltent principalement dans les jachères alors que celles à usage thérapeutique se rencontrent en savanes arborées et forêts claires. Les forêts galeries abritent les espèces à usages multiples. La destruction de ces écosystèmes naturels accentue la perte de la biodiversité végétale.

() 2016 International Formulae Group. All rights reserved.

Mots clés : Biodiversité floristique, valeur d'usage, zone soudanienne, Côte d'Ivoire

\section{Flora biodiversity and value in use in the Sudan region of Cote d'Ivoire:}

\begin{abstract}
The vegetation of northern Côte d'Ivoire is mostly dominated by the savannahs that have been subject of little botanical studies. The present study conducted at Tengrela in northern Côte d'Ivoire was to assess the diversity of plant vegetation and their uses by local populations. Data collection combined ground record and the itinerant survey. Ethnobotanical surveys were conducted with traditional health practitioners to understand the cultural significance of species. The study highlighted six vegetations that were woodlands, gallery forests,
\end{abstract}


fallows, wooded grasslands, ponds and grasslands. There were 402 species distributed into 269 genera and 84 families including 22 threatened species. The survey reveals that many plant species were used by local people for food, disease treating, and various other uses. Food use species were found mainly in fallows while those for therapeutic use were found in wooded savannahs and woodlands. Plant species with various uses were found mainly in gallery forests. The destruction of these natural ecosystems emphasizes the loss of plant biodiversity.

(C) 2016 International Formulae Group. All rights reserved.

Keywords: Floristic biodiversity, use value, Sudan region, Côte d'Ivoire.

\section{INTRODUCTION}

Plusieurs travaux scientifiques ont abordé la question de la richesse et de la composition floristique des écosystèmes naturels et indexés les activités anthropiques comme les principales causes de la déforestation (Aké-Assi, 2001; Aké-Assi, 2002; Adou Yao et N'Guessan, 2005; Vroh, 2013). Au cours de la période 1990 à 2005, une estimation moyenne donne un taux de déforestation de 20,7\% pour l'Afrique de l'Ouest avec une légère augmentation pour la Côte d'Ivoire (FAO, 2006). Cette régression du couvert végétal est due à l'essor de l'exploitation des espèces de bois d'œuvre (Barima et al., 2009). De plus, les pratiques culturales et l'urbanisation sont aussi des causes importantes de déforestation (MINEEF, 2007 ; Konan, 2008).

En zone soudanienne, au nord de la Côte d'Ivoire, la culture de l'anacarde occupe des surfaces de plus en plus importantes (Dugué et al., 2003). Cette économie agricole est soutenue par une forte croissance démographique. En effet, la démographie constitue un facteur prépondérant de l'intensification des défrichements agricoles, donc de la dégradation des terres (Gafsi, 2007). Si le développement des cultures industrielles et vivrières est l'une des causes responsables de la disparition du couvert végétal, l'exploitation minière (or, manganèse et nickel) n'en demeure pas moins un facteur avancé de dégradation de la flore de Côte
d'Ivoire (N'Guessan, 2010, 2012 ; Vroh et al., 2014). La destruction des écosystèmes naturels s'accompagne d'une perte de la diversité biologique (Teyssèdre, 2004). Des espèces animales et végétales liées à certains biotopes sont directement menacées de disparition (Lavergne, 2004). Il importe donc de faire un état des lieux de la flore et de la végétation de la Côte d'Ivoire (Koffi et al., 2015).

A ce jour, les études entreprises sur la richesse et la diversité floristique de la Côte d'Ivoire se sont concentrées dans la partie Sud forestière du pays (N'Da et al., 2008 ; Adou Yao et N'Guessan, 2005 ; Kassi et al., 2010 ; Koffi et al., 2015). Le Nord de la Côte d'Ivoire, savanicole, a été peu exploré (Ambé, 2001; Koffi et al., 2014). Des études additionnelles doivent être menées pour estimer la diversité floristique du Nord de la Côte d'Ivoire, faire un état des lieux des différentes formations végétales rencontrées dans cette région et évaluer la valeur écologique de ces formations végétales pour le bien-être des populations locales (Koffi et al., 2015).

La présente étude se propose de contribuer à une meilleure connaissance de la flore et de la végétation du Nord savanicole de la Côte d'Ivoire. De façon spécifique, il s'agit de quantifier la richesse et la composition spécifique, de caractériser la végétation, d'identifier les usages faits des plantes par les populations locales et d'examiner la 
disponibilité des plantes utiles selon les types de végétation rencontrés.

\section{MATERIEL ET METHODES}

\section{Site d'étude}

La zone, objet de la présente étude, est située dans le Nord de la Côte d'Ivoire dans le Département de Tengrela (Figure 1). Le département de Tengrela a pour coordonnées géographiques $783755 \mathrm{~N}$ et $1159746 \mathrm{~W}$. Le climat est de type tropical. La température moyenne annuelle est de $27,5^{\circ} \mathrm{C}$. Il tombe en moyenne $1080 \mathrm{~mm}$ de pluie par an. La végétation de la région est de type soudanais (Guillaumet et Adjanohoun, 1971). Elle se présente comme l'association de forêts claires et de savanes (Amon et al., 2015) se développant sur des sols ferralitiques moyennement désaturés et moins développés. Ces sols présentent des phénomènes d'induration faibles et irréguliers. L'horizon gravillonnaire est plus important. On y relève la présence de nombreuses zones de cuirasses (Perraud, 1971).

\section{Collecte des données}

Deux méthodes de collecte ont été utilisées. Il s'agit du relevé de surface et de l'inventaire itinérant (Adou Yao et N'Guessan, 2005). Ces deux types de relevés sont complémentaires. Le relevé de surface a consisté à délimiter une parcelle de $10 \mathrm{~m}$ x 10 $\mathrm{m}\left(100 \mathrm{~m}^{2}\right)$ dans les différents types de formations végétales afin de recenser le maximum d'espèces végétales.

A l'intérieur de cette surface de 100 $\mathrm{m}^{2}$, toutes les espèces de plantes rencontrées sont identifiées et leurs noms sont notés sur la fiche de relevé portant le numéro de la parcelle. Les coordonnées géographiques de la parcelle sont enregistrées à l'aide d'un GPS. Les espèces non identifiées sont prélevées pour la confection d'un herbier et identifiées ultérieurement en Laboratoire. Les inventaires itinérants ou relevé de parcours ont été essentiellement faits sur des layons dans le sens Nord-Sud et Est-Ouest de la zone d'étude. Toutes les espèces de plantes qui n'avaient pas été rencontrées dans les placettes ont été notées.

Des interviews ont été réalisées auprès des populations locales notamment les tradipraticiens pour appréhender l'importance culturelle des espèces végétales. Les données sur l'utilisation des espèces végétales dans les divers domaines traditionnels ont été recueillies. Cet aspect du travail est important d'autant plus que l'utilisation des espèces par les populations locales est un facteur de destruction des peuplements sauvages mais aussi un moyen de conservation de certaines espèces (Agbahungba et al., 2001).

\section{Analyse des données}

Les résultats bruts ont fait l'objet de deux types d'analyses, qualitatives et quantitatives. Les analyses qualitatives ont consisté à dénombrer le nombre total d'espèces, de genres et de familles, la chorologie et/ou le statut écologique de chaque espèce. Les espèces à statut particulier (espèces endémiques et espèces rares et/ou en voie de disparition) ont été identifiées en se basant d'une part sur leurs affinités chorologiques et d'autre part sur les liste de l'UICN (2015) et de Aké-Assi (1988). L'abondance des espèces a été évaluée à travers leurs fréquences d'apparition dans les placettes.

La diversité quantitative a été évaluée par le calcul des indices tels que l'indice de diversité de Shannon et Weaver (1948), l'indice d'équitabilité de Piélou (1966) et le coefficient de similitude de Sørensen (1948) afin d'évaluer la richesse floristique, la diversité spécifique et l'équitabilité floristique du site et la ressemblance des biotopes. En désignant par $N$ la fréquence totale des $S$ espèces considérées, $n i$ la fréquence d'une espèce $i$ et $P i(n i / N)$ la fréquence relative de 
l'espèce $i$, l'indice de Shannon $(H)$ se résume à l'expression mathématique suivante :

$$
H=-\sum_{i=1}^{s} P i \times \ln P i
$$

Cet indice varie de 0 (une seule espèce présente) à $\log _{2} \mathrm{~S}$ (toutes les espèces présentes ont une même abondance). L'équitabilité $(E)$ s'obtient en rapportant la diversité observée à la diversité théorique maximale. L'équitabilité varie de 0 à 1 . Elle tend vers 0 quand la quasitotalité des effectifs est concentrée sur une espèce et vers 1 lorsque toutes les espèces ont la même abondance. Dans le cas où cet indice tend vers 1, le milieu en question est dit équilibré. L'indice d'équitabilité $(E)$ se calcule selon la formule mathématique suivante :

$$
E=\frac{H}{\ln S}
$$

Le coefficient de similitude de Sørensen $(K s)$ représente la diversité $\beta$ qui est le taux de remplacement des espèces et individus lors du passage d'un site à un autre. Elle a pour but de caractériser objectivement et quantitativement le degré de ressemblance de deux relevés ou biotopes. (Condit et al., 2002). Il se calcule de la manière suivante :

$$
K s=\frac{2 c}{a+b} \times 100
$$

Dans cette formule, Ks représente le coefficient de similitude de Sørensen ; a le nombre d'espèces d'une liste appartenant à un site $\mathrm{A}$; $\mathrm{b}$ le nombre d'espèces d'une liste appartenant à un site $\mathrm{B}$ et $\mathrm{c}$ le nombre d'espèces communes aux deux sites (A et B) que l'on veut comparer. Ce coefficient varie entre 0 et $100 \%$. L'hypothèse de similitude ou ressemblance est admise, lorsque le coefficient est supérieur à $50 \%(K s>50)$. Dans le cas contraire $(K s<50)$, il n'y a pas de similitude entre les listes floristiques des parcelles concernées (Troupin, 1966).
Des analyses de variance à un facteur (ANOVA 1) ou des tests non paramétriques de Kruskal-Wallis ont permis de comparer les valeurs moyennes des indices de diversité des différents biotopes. Le dernier test a été réalisé pour la comparaison des valeurs moyennes des indices d'équitabilité dont les données présentaient des variances inégales et non normalisées. La signification du test est déterminée en comparant la probabilité $P$ associée à la statistique du test au seuil $\alpha=$ 0,05 . Lorsque la différence analysée est significative, il est procédé à la comparaison des moyennes. Le logiciel XLSTAT version 2014.5.03 a été utilisé pour la réalisation de ces tests.

Par la suite, les éventuelles relations entre les espèces, leurs usages et les biotopes ont été vérifiées à travers une analyse des correspondances multiples (ACM). L'ACM est une extension de l'analyse factorielle des correspondances (AFC) et consiste simplement à réaliser l'AFC du tableau de Burt établis. C'est une méthode statistique descriptive qui a pour objet l'étude simultanée de plus de deux caractères. Elle est utilisée le plus souvent dans la description des tableaux de données des résultats d'enquêtes auprès des personnes. Ces données de résultats sont présentées dans un tableau de Burt. Les lignes correspondent alors aux individus enquêtés et les colonnes correspondent aux diverses modalités de réponses des questions posées. Le but est de comprendre les liaisons pouvant exister entre les variables considérées. On aboutit à des cartes de représentation sur lesquelles on peut observer les proximités entre les catégories des variables qualitatives et les observations (Chardon, 1981 ; Escofier et Pagès, 1989). Les variables considérées dans le cadre de cette étude sont les espèces citées par les populations, leurs usages potentiels (alimentaires, médicinales et divers usages) et les biotopes de récolte. Le logiciel 
$\mathrm{R}$ version 3.1 .3 a été utilisé pour la réalisation de ce test.

\section{RESULTATS}

\section{Diversité qualitative de la flore du site}

L'étude de la flore a permis de recenser 6 formations végétales. Ce sont les forêts claires, les galeries forestières, les jachères, les savanes arborées/arbustives, les mares et les savanes herbeuses. La richesse floristique est estimée à 402 espèces de plantes. Elles se répartissent en 269 genres et 84 familles. Soixante-dix (70) genres comprennent au moins deux (2) espèces et 199 genres ne contiennent qu'une seule espèce. La plupart des genres recensés dans les placettes sont donc représentés par une seule espèce. Les genres les plus représentés sont: Ficus (12 espèces), Combretum (9 espèces), Hyparrhenia et Panicum (6 espèces chacun). Les familles les plus riches en espèces sont celles des Poaceae (61 espèces), des Fabaceae (31 espèces), des Euphorbiaceae (27), des Caesalpiniaceae (19) espèces), des Combretaceae et des Rubiaceae (17 espèces chacune). Douze (12) familles sur les 84 comprennent au moins 10 espèces chacune.

Les espèces les plus prépondérantes du site d'étude selon leur fréquence d'apparition dans les placettes sont Baissea multiflora A. DC (29 relevés), Lannea acida A. Rich (24 relevé) et Parkia biglobosa (Jacq.) Benth. (24 relevés). Les cinq (5) espèces suivantes dont les fréquences varient entre 18 et 19 sont Annona senegalensis Pers. (19 relevés), Daniellia oliveri Hutch. et Dalz. (19 relevés), Isoberlinia doka Craib ex Stapf (19 relevés), Gardenia ternifolia Schum. et Thonn. et Securinega virosa (Roxb. ex Willd.) Baillon avec 18 relevés chacune. Parmi les espèces les plus prépondérantes (fréquences d'apparition dans les placettes supérieures à 12), seulement 3 sont des lianes. Très peu de lianes dominent dans la flore du site. Dans l'ensemble des espèces recensées, $21,14 \%$ sont typiquement forestières (Guineo-Congolaises) et 34,57\% sont strictes des savanes soudanaises (Soudano-Zambéziennes). Il faut aussi noter une forte proportion des espèces de la zone de transition forêt-savane (Guineo-Congolaises et Soudano-Zambéziennes).

La liste générale des espèces recensées révèle la présence de 22 espèces à statut particulier ou à forte valeur pour la conservation, soit $5,47 \%$ de l'ensemble des espèces (Tableau 1). Parmi ces espèces, 6 sont citées comme rares et menacées d'extinction de la flore ivoirienne selon Aké-Assi (1998). Dans cette catégorie, une espèce Uvaria tortilis A. Chev. est endémique de la Côte d'Ivoire. Seize (16) espèces se retrouvent sur la liste rouge de l'UICN (2015). Dans ce groupe, on enregistre 3 espèces classées comme vulnérables, 12 espèces à préoccupation mineure et une espèce classée à la fois à risque faible et à préoccupation mineure (Tableau 1).

\section{Diversité quantitative de la flore du site}

L'indice de Shannon est de 4,72 $\pm 0,89$ dans les forêts claires. La plus faible valeur de cet indice est observée dans les savanes herbeuses $(2,98 \pm 1,33)$. Les autres biotopes ont leurs indices compris entre ces deux valeurs extrêmes (Tableau 2). D'un biotope à un autre, il existe une différence significative $(F=3,38 ; P=0,006)$. Les plus grandes valeurs d'indice d'équitabilité de Piélou sont observées dans les mares et les savanes herbeuses. Ces valeurs sont identiques et égales à $0,99 \pm 0,01$. La plus faible valeur est enregistrée au niveau des forêts claires (Tableau 2). Le test de Kruskal-Wallis indique qu'il existe une différence significative entre ces valeurs moyennes $\left(\chi^{2}=16,39 ; P=0,022\right)$. Le coefficient de similitude de Sørensen montre qu'il n'y a pas de ressemblance floristique entre les différents biotopes. Ainsi, chaque biotope est caractérisé par un cortège floristique spécifique. 


\section{Valeur d'usage}

Plusieurs usages sont associés aux plantes récoltées. Ce sont l'usage thérapeutique, alimentaire et autres (artisanal, construction, bois énergie, etc.). Sur l'ensemble des espèces collectées, 77 ont été identifiées par les populations riveraines comme plantes utilitaires (Tableau 3). L'usage thérapeutique est le plus observé dans la zone d'étude. Ce sont 50,44\% des espèces citées qui sont concernées par ce type d'usage. Elles sont utilisées pour traiter différentes pathologies. Les traitements les plus courants concernent l'accès fébrile (Alchornea cordifolia, Annona senegalensis, Adansonia digitata etc.) l'entéralgie (Cussonia barteri, Holarrhena floribunda, Khaya senegalensis, Syzygium guineense var macrocarpum...), la cicatrisation des blessures (Hymenocardia acida), les hémorroïdes (Albizia ferruginea). Ces plantes sont aussi utilisées comme revigorants (Cryptolepsis sanguinolenta), ou aphrodisiaques (Paullinia pinnata, secamone afzelii, Clausena anisata). Les espèces alimentaires représentent $20,35 \%$ des espèces citées par les populations locales. Les plantes alimentaires citées par les populations le sont pour la plupart pour leurs fruits comestibles (Nauclea latifolia, Parinari curatellifolia, Parkia biglobosa, Pouteria alnifolia, Pterocarpus santalinoides, Saba senegalensis, Uvaria chamae, Vitex doniana, Ximenia Americana...). Les espèces fruitières représentent $77,77 \%$ de l'ensemble des espèces alimentaires citées. D'autres espèces le sont parce qu'elles entrent dans la confection de divers repas (Elaeis guineensis, Lophira lanceolata, Ceiba pentandra, Adansonia digitata...). Les parties consommées sont les feuilles, les fruits, les tiges, les rhizomes et aussi la sève extraite en vin. Trente-trois espèces $(29,20 \%)$ sont utilisées par les populations locales pour divers besoins. Il s'agit de plantes entrant dans la construction des habitats. Plusieurs autres sont également utilisées pour le façonnement d'objet d'art, l'ornementation, la construction de mobilier domestique.

\section{Disponibilité des espèces utilitaires}

L'Analyse des Correspondances Multiples a permis de discriminer 3 groupes d'espèces en relation avec les biotopes de récolte et les usages (Figure 2). Les deux premiers axes de l'ACM expliquent 43,06\% de la variabilité. L'axe 1 est lié aux différents usages tandis que l'axe 2 oppose les espèces de forêts galeries contre celles rencontrées dans les forêts claires, les savanes arborées et les jachères. Le groupe 1 comptent les espèces qui se rencontrent en abondance dans les jachères et reconnues par les populations locales comme compléments alimentaires. Les plus citées par celles-ci sont Ceiba pentandra (Linn.) Gaerth., Elaeis guineensis Jacq. ; Vittelaria paradoxa Gaertn. F., Abelmoshus esculentus, Ximenia americana Linn., Uvaria chamae P. Beauv. et Nauclea latifolia Smith.

Le deuxième groupe d'espèces se retrouve dans les savanes arborées et les forêts claires et sont considérées comme des espèces ayant un usage thérapeutique. Il s'agit de Diospyros mespiliformis Hochst. ex A. DC., Cochlospermum planchonii Hook.f., Khaya senegalensis (Desv.) A. Juss., Bombax costatum Pellegr et Bauhinia thonningii. Le troisième groupe cité par les populations locales est celui des espèces à usages multiples recensées dans les forêts galeries. Ce sont les espèces telles que Afzelia africana Sm., Anogeissus leiocarpus (DC.) Guill. et Perr., Antiaris toxicaria var. africana (Engl.) C.C. Berg, Berlinia grandiflora (Vahl) Hutch. et Dalz., Cassia sieberiana DC., Lophira lanceolata Tiegh. ex Keay, Parinari curatellifolia Planchon ex Benth. 


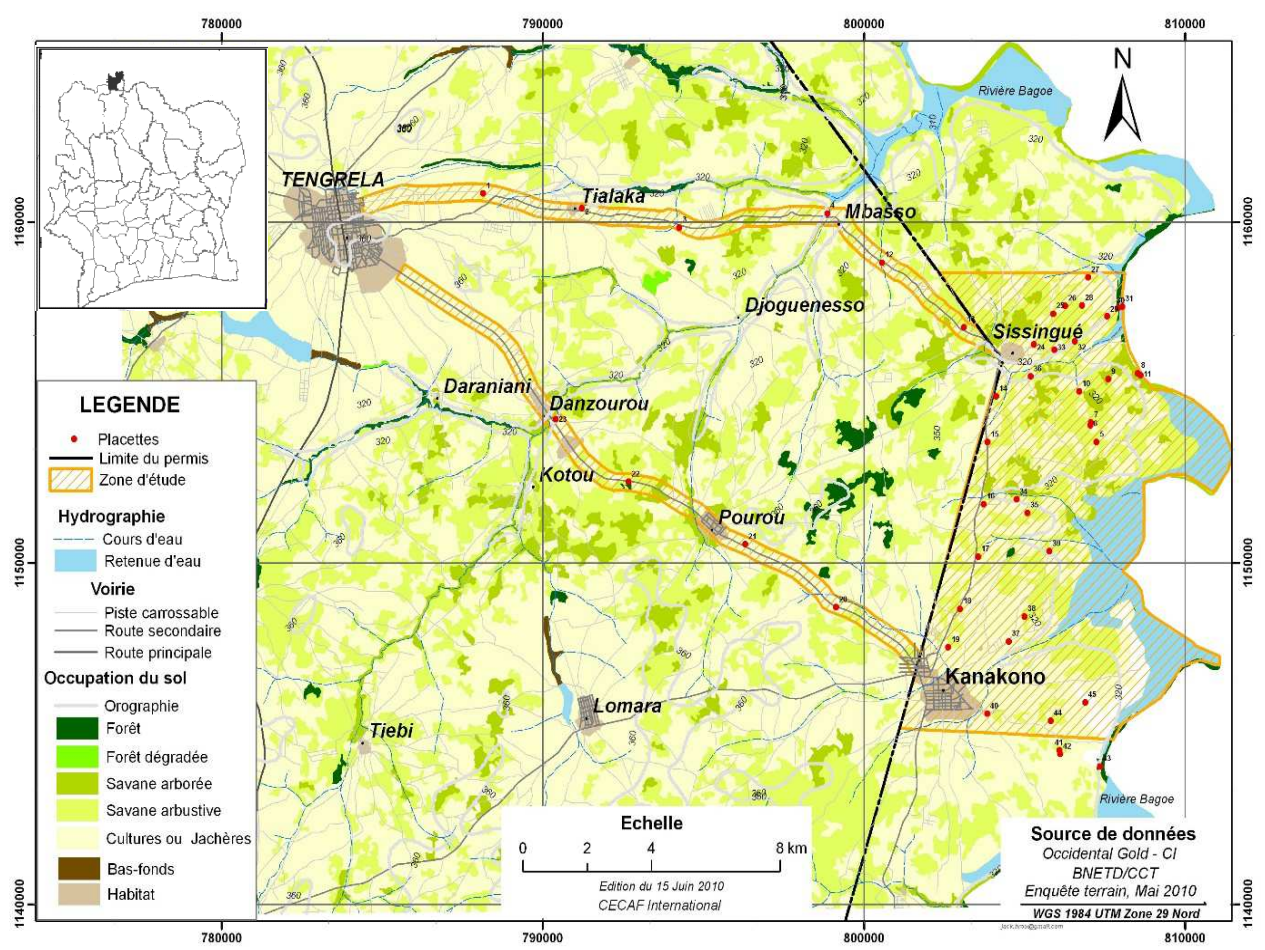

Figure 1 : Carte de localisation de la zone d'étude (région de Tengrela).

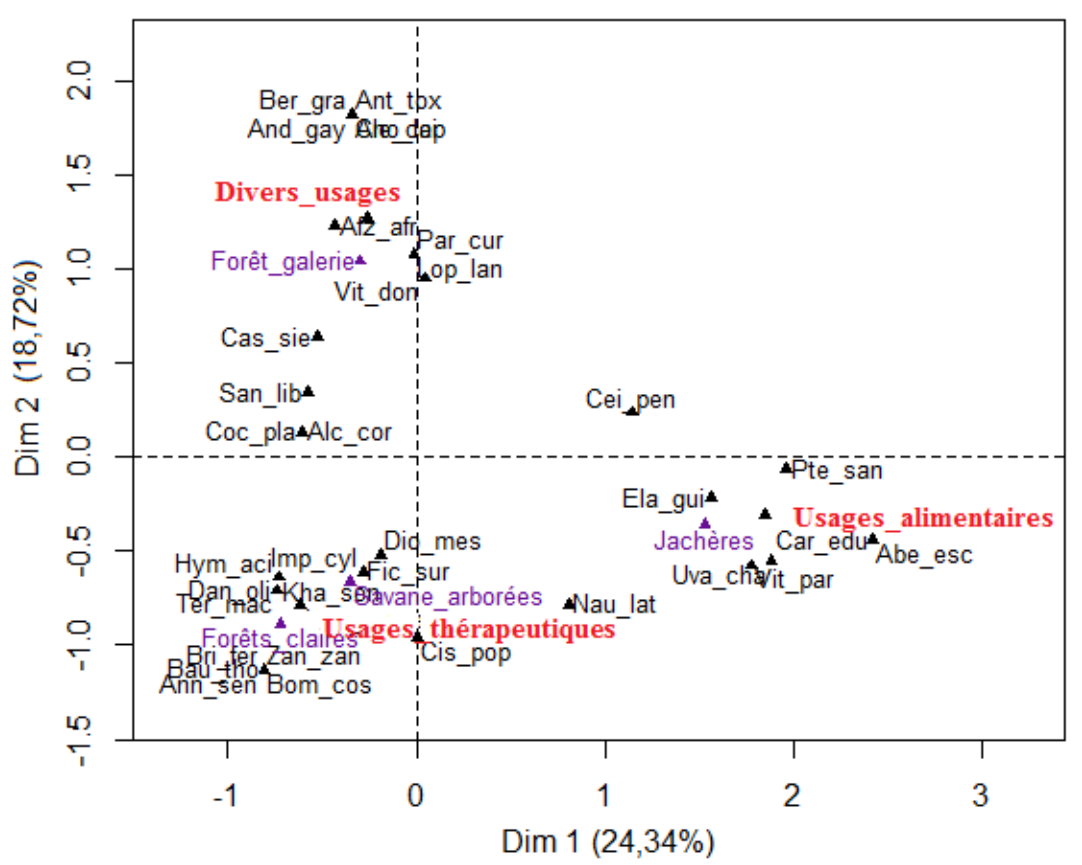

Figure 2 : Discrimination des plantes utilitaires selon les biotopes dans la région de Tengrela. 
D. OUATTARA et al. / Int. J. Biol. Chem. Sci. 10(3): 1122-1138, 2016

Tableau 1 : Espèces rares et menacées d'extinction rencontrées à Tengrela.

\begin{tabular}{|c|c|c|c|c|c|c|}
\hline $\mathbf{N}^{\circ}$ & Espèces & Famille & TB & Choro & UICN (2015) & Aké-Assi (1998) \\
\hline 1 & Afzelia africana $\mathrm{Sm}$. & Caesalpiniaceae & $\mathrm{mP}$ & GC-SZ & $\mathrm{Vu}$ & \\
\hline 2 & Aspilia helianthoides subsp. ciliata (Schum.) Adams & Asteraceae & np & $\mathrm{SZ}$ & LC & \\
\hline 3 & Cissus doeringii Gilg \& Brandt & Vitaceae & $\mathrm{H}$ & GC-SZ & $\mathrm{LC}$ & \\
\hline 4 & Cleidion gabonicum Baill. & Euphorbiaceae & $\mathrm{mp}$ & $\mathrm{GC}$ & & Rare \\
\hline 5 & Croton membranaceus Müll. Arg. & Euphorbiaceae & np & GC & & Rare \\
\hline 6 & Cyperus distans Linn. F. & Cyperaceae & $\mathrm{H}$ & GC-SZ & $\mathrm{LC}$ & \\
\hline 7 & Detarium microcarpum Guill. et Perr. & Caesalpiniaceae & $\mathrm{mp}$ & $\mathrm{SZ}$ & $\mathrm{LC}$ & \\
\hline 8 & Dicliptera elliotii C.B. Cl. & Acanthaceae & $\mathrm{Ch}$ & GC-SZ & $\mathrm{LC}$ & \\
\hline 9 & Echinochloa colona (Linn.) Link. & Poaceae & Th & GC-SZ & $\mathrm{LC}$ & \\
\hline 10 & Echinochloa pyramidalis (Lam.) Hitchc. et Chase & Poaceae & H (Hyd) & GC-SZ & LC & \\
\hline 11 & Elionorus platypus Hack. & Poaceae & $\mathrm{H}$ & SZ & $\mathrm{LC}$ & \\
\hline 12 & Fimbristylis dichotoma $\mathrm{Vahl}$ & Cyperaceae & $\mathrm{H}$ & GC-SZ & LC & \\
\hline 13 & Khaya senegalensis (Desv. A. Juss. & Meliaceae & $\mathrm{mP}$ & SZ & $\mathrm{Vu}$ & \\
\hline 14 & Lannea nigritana (Sc. Elliot) Keay & Anacardiaceae & $\mathrm{mp}$ & GC-SZ & & Rare \\
\hline 15 & Nauclea xanthoxylon (A. Chev.) Aubrév. & Rubiaceae & $\mathrm{mP}$ & GC & & Rare \\
\hline 16 & Paspalum scrobiculatum Linn. & Poaceae & $\mathrm{H}$ & GC-SZ & LC & \\
\hline 17 & Pterocarpus santalinoides L'Hérit. ex DC. & Fabaceae & $\mathrm{mp}$ & GC-SZ & $\mathrm{LR} / \mathrm{LC}$ & \\
\hline 18 & Rhynchosia minima (Linn.) DC. var. memnonia DC. & Fabaceae & Lnp & GC-SZ & LC & \\
\hline 19 & Syzygium gü̈neense (Willd.) DC. var. guineense & Myrtaceae & $\mathrm{mp}$ & GC & & Rare \\
\hline 20 & Tacca IeontopetaIoides (Linn.) O. Ktze & Taccaceae & $\mathrm{G}$ & SZ & LC & \\
\hline 21 & Uvaria tortilis A. Chev. & Annonaceae & Lmp & GCi & & Rare \\
\hline 22 & Vittelaria paradoxa Gaertn. F. & Sapotaceae & $\mathrm{mp}$ & SZ & $\mathrm{Vu}$ & \\
\hline
\end{tabular}

TB : Type biologique ; Choro : Chorologie ; LC : Préoccupation mineure ; LR : Risque faible ; Vu : Vulnérable ; GC: Taxon de la région Guinéo-Congolaise ; GCi: Taxon endémique à la Côte d'Ivoire ; SZ : Taxon de la région Soudano-Zambézienne ; GC-SZ: Taxon de la zone de transition entre les régions Guinéo-Congolaise et Soudano-Zambézienne ; G : Géophyte ; H: Hémicryptophyte ; Th: Thérophyte ; Ch : Chaméphyte (taxon dont la hauteur est comprise entre 0 et $0,25 \mathrm{~m}$ ) ; np: Nanophanérophyte (taxon dont la hauteur est comprise entre 0,25 et $2 \mathrm{~m}$ ) ; mp: Microphanérophyte (taxon dont la hauteur est comprise entre 2 et $8 \mathrm{~m}$ ) ; mP: Mésophanérophyte (taxon dont la hauteur est comprise entre 8 et $32 \mathrm{~m}$ ) ; L : liane ; Hyd : Hydrophyte. 
D. OUATTARA et al. / Int. J. Biol. Chem. Sci. 10(3): 1122-1138, 2016

Tableau 2 : Indices de diversité de Shannon et d'Equitabilité de Piélou des types de végétation rencontrés à Tengrela.

\begin{tabular}{|c|c|c|c|c|c|c|c|}
\hline Variable & Forêt claire & $\begin{array}{c}\text { Galerie } \\
\text { forestière }\end{array}$ & Jachère & $\begin{array}{c}\text { Savane } \\
\text { arborée/arbustive }\end{array}$ & Mare & $\begin{array}{c}\text { Savane } \\
\text { herbeuse }\end{array}$ & $\begin{array}{c}\text { Statistiques du } \\
\text { test }\end{array}$ \\
\hline $\begin{array}{l}\text { Indice }(\mathrm{H}) \text { de Shannon } \\
\text { et Weaver }\end{array}$ & $4,72 \pm 0,89^{b}$ & $4,45 \pm 0,83^{\mathrm{ab}}$ & $4,42 \pm 0,83^{a b}$ & $4,13 \pm 0,71^{\mathrm{ab}}$ & $3,66 \pm 0,93^{\mathrm{ab}}$ & $2,98 \pm 1,33^{\mathrm{a}}$ & $\begin{array}{l}F=3,38 \\
P=0,006\end{array}$ \\
\hline $\begin{array}{l}\text { Equitabilité (E) de } \\
\text { Piélou }\end{array}$ & $0,95 \pm 0,03^{\mathrm{ab}}$ & $0,97 \pm 0,02^{\mathrm{ab}}$ & $0,98 \pm 0,01^{\mathrm{b}}$ & $0,98 \pm 0,01^{\mathrm{b}}$ & $0,99 \pm 0,01^{\mathrm{b}}$ & $0,99 \pm 0,01^{\mathrm{b}}$ & $\begin{aligned} \chi^{2} & =16,39 \\
P & =0,022\end{aligned}$ \\
\hline
\end{tabular}

Tableau 3 : Valeur d'usage des espèces citées par les populations locales.

\begin{tabular}{|c|c|c|c|c|c|}
\hline $\mathbf{N}^{\circ}$ & Espèces & Familles & Divers usages & Plante alimentaire & Plante médicinale \\
\hline 1 & Acacia pennata & Mimosaceae & $\mathrm{x}$ & & \\
\hline 2 & Adansonia digitata Linn. & Bombacaceae & $\mathrm{x}$ & $\mathrm{x}$ & $\mathrm{x}$ \\
\hline 3 & Afzelia africana $\mathrm{Sm}$. & Caesalpiniaceae & $\mathrm{x}$ & & $\mathrm{x}$ \\
\hline 4 & Albizia ferruginea (Guill. \& Perr.) Benth. & Mimosaceae & & & $\mathrm{x}$ \\
\hline 5 & Alchornea cordifolia (Schum. \& Thonn.) Müll.Arg. & Euphorbiaceae & $\mathrm{x}$ & & $\mathrm{x}$ \\
\hline 6 & Andropogon gayanus Kunth var. gayanus & Poaceae & $\mathrm{x}$ & & \\
\hline 7 & Annona senegalensis Pers. & Annonaceae & & & $\mathrm{x}$ \\
\hline 8 & Anogeissus leiocarpus (DC.) Guill. \& Perr. & Combretaceae & $\mathrm{x}$ & & \\
\hline 9 & Antiaris toxicaria var. africana (Engl.) C.C. Berg & Moraceae & $\mathrm{x}$ & & \\
\hline 10 & Bauhinia thonningii Linn & Caesalpiniaceae & & & $\mathrm{x}$ \\
\hline 11 & Berlinia grandiflora (Vahl) Hutch. \& DaIz. & Caesalpiniaceae & $\mathrm{x}$ & & \\
\hline 12 & Bombax costatum Pellegr. \& Vuillet & Bombacaceae & & & $\mathrm{x}$ \\
\hline
\end{tabular}


D. OUATTARA et al. / Int. J. Biol. Chem. Sci. 10(3): 1122-1138, 2016

\begin{tabular}{|c|c|c|c|c|c|}
\hline 13 & Bridelia ferruginea Benth. & Euphorbiaceae & & & $\mathrm{x}$ \\
\hline 14 & Carissa edulis Vahl & Apocynaceae & & $\mathrm{x}$ & \\
\hline 15 & Cassia sieberiana DC. & Caesalpiniaceae & $\mathrm{x}$ & & $\mathrm{x}$ \\
\hline 16 & Ceiba pentandra (Linn.) Gaerth. & Bombacaceae & $\mathrm{x}$ & $\mathrm{x}$ & $\mathrm{x}$ \\
\hline 17 & Cissus populnea Guill. \& Perr. & Vitaceae & & $\mathrm{x}$ & $\mathrm{x}$ \\
\hline 18 & Clausena anisata (Willd.) Benth. & Rutaceae & & & $\mathrm{x}$ \\
\hline 19 & Clerodendrum capitatum (Willd.) Schum. \& Thonn. var. capitalum & Verbenaceae & $\mathrm{x}$ & & \\
\hline 20 & Cochlospermum planchonii Hook.f. & Cochlospermaceae & $\mathrm{x}$ & & $\mathrm{x}$ \\
\hline 21 & Combretum molle R. Br. ex G. Don & Combretaceae & & & $\mathrm{x}$ \\
\hline 22 & Combretum paniculatum Vent. & Combretaceae & $\mathrm{x}$ & & \\
\hline 23 & Crossopteryx febrifuga (G. Don) Benth. & Rubiaceae & & & $\mathrm{x}$ \\
\hline 24 & Cryptolepis sanguinolenta (Lindl.) Schltr. & Periplocaceae & & & $\mathrm{x}$ \\
\hline 25 & Cussonia barteri Linn. & Araliaceae & & & $\mathrm{x}$ \\
\hline 26 & Daniellia olivera Hutch. \& Dalz. & Caesalpiniaceae & $\mathrm{x}$ & & $\mathrm{x}$ \\
\hline 27 & Detarium microcarpum Guill. \& Perr. & Caesalpiniaceae & & & $\mathrm{x}$ \\
\hline 28 & Dichrostachys cinerea (Linn.) Wight \& Arn. subsp. Cinerea & Mimosaceae & & & $\mathrm{x}$ \\
\hline 29 & Diospyros mespiliformis Hochst. ex A. DC. & Ebenaceae & $\mathrm{x}$ & $\mathrm{x}$ & $\mathrm{x}$ \\
\hline 30 & Elaeis guineensis Jacq. & Arecaceae & $\mathrm{x}$ & $\mathrm{x}$ & $\mathrm{x}$ \\
\hline 31 & Erythrina senegalensis DC. & Fabaceae & $\mathrm{x}$ & & $\mathrm{x}$ \\
\hline 32 & Euphorbia hirta Linn. & Euphorbiaceae & & & $\mathrm{x}$ \\
\hline 33 & Ficus sur Forsk. & Moraceae & $\mathrm{x}$ & $\mathrm{x}$ & $\mathrm{x}$ \\
\hline 34 & Ficus vallis-choudae Del. & Moraceae & & $\mathrm{x}$ & \\
\hline 35 & Flacourtia flavescens Willd. & Flacourtiaceae & & $\mathrm{x}$ & \\
\hline 36 & Gardenia erubescens Stapf \& Hutch. & Rubiaceae & & $\mathrm{x}$ & \\
\hline
\end{tabular}


D. OUATTARA et al. / Int. J. Biol. Chem. Sci. 10(3): 1122-1138, 2016

\begin{tabular}{|c|c|c|c|c|c|}
\hline 37 & Holarrhena floribunda (G. Don) Dur. \& Schinz var. floribunda & Apocynaceae & & & $\mathrm{x}$ \\
\hline 38 & Hymenocardia acida Tul. & Euphorbiaceae & $\mathrm{x}$ & & $\mathrm{x}$ \\
\hline 39 & Imperata cylindrica Linn & Poaceae (Gramineae) & $\mathrm{x}$ & $\mathrm{x}$ & $\mathrm{x}$ \\
\hline 40 & Khaya senegalensis (Desv.) A. Juss. & Meliaceae & $\mathrm{x}$ & & $\mathrm{x}$ \\
\hline 41 & Lecaniodiscus cupanioides Planch. & Sapindaceae & & $\mathrm{x}$ & \\
\hline 42 & Lonchocarpus cyanescens (Schummach \& Thonn.) Benth. & Fabaceae & $\mathrm{x}$ & & \\
\hline 43 & Lophira lanceolata van Tiegh. ex Keay & Ochnaceae & $\mathrm{x}$ & $\mathrm{x}$ & $\mathrm{x}$ \\
\hline 44 & Manilkara multinervis (Bak.) Dubard & Sapotaceae & $\mathrm{x}$ & & \\
\hline 45 & Margaritaria discoidea ( Baill .) Webster & Euphorbiaceae & & & $\mathrm{x}$ \\
\hline 46 & Maytenus senegalensis (Lam.) Exell & Celastraceae & & & $\mathrm{x}$ \\
\hline 47 & Mitragyna inermis (Willd.) Kuntze & Rubiaceae & & & $\mathrm{x}$ \\
\hline 48 & Morinda lucida Benth. & Rubiaceae & & & $\mathrm{x}$ \\
\hline 49 & Nauclea latifolia $\mathrm{Sm}$. & Rubiaceae & & $\mathrm{x}$ & $\mathrm{x}$ \\
\hline 50 & Parinari curatellifolia Planch. ex Benth. & Chrysobalanaceae & $\mathrm{x}$ & $\mathrm{x}$ & $\mathrm{x}$ \\
\hline 51 & Parkia biglobosa (Jacq.) Benth. & Mimosaceae & $\mathrm{x}$ & $\mathrm{x}$ & $\mathrm{x}$ \\
\hline 52 & Paullinia pinnata $\mathrm{L}$. & Sapindaceae & & & $\mathrm{x}$ \\
\hline 53 & Phyllanthus muellerianus (O. Ktze.) Exell & Euphorbiaceae & $\mathrm{x}$ & & \\
\hline 54 & Pouteria alnifolia (Bak.) Roberty & Sapotaceae & & $\mathrm{x}$ & \\
\hline 55 & Pterocarpus erinaceus Poilr. & Fabaceae & $\mathrm{x}$ & & \\
\hline 56 & Pterocarpus santalinoides L'Hérit. ex DC. & Fabaceae & $\mathrm{x}$ & $\mathrm{x}$ & \\
\hline 57 & Saba senegalensis var. glabriflora (Hua) Pichon & Apocynaceae & & $\mathrm{x}$ & \\
\hline 58 & Sansevieria liberica Gérôme \& Labr. & Agavaceae & $\mathrm{x}$ & & $\mathrm{x}$ \\
\hline 59 & Secamone afzelii (Schultes) K. Schum. & Asclepiadaceae & & & $\mathrm{x}$ \\
\hline 60 & Securidaca longepedunculata Fresen. & Polygalaceae & & & $\mathrm{x}$ \\
\hline
\end{tabular}


D. OUATTARA et al. / Int. J. Biol. Chem. Sci. 10(3): 1122-1138, 2016

\begin{tabular}{|c|c|c|c|c|c|}
\hline 61 & Securinega virosa Linn. & Polygalaceae & & & $\mathrm{x}$ \\
\hline 62 & Smilax kraussiana Meisn. & Smilacaceae & & & $\mathrm{x}$ \\
\hline 63 & Sterculia setigera Del. & Sterculiaceae & & & $\mathrm{x}$ \\
\hline 64 & Syzygium guineense var. macrocarpum EngI. & Myrtaceae & & & $\mathrm{x}$ \\
\hline 65 & Tamarindus indica Linn. & Caesalpiniaceae & & & $\mathrm{x}$ \\
\hline 66 & Tapinanthus bangwensis (Engl. \& K. Krause) Danser & Loranthaceae & & & $\mathrm{x}$ \\
\hline 67 & Terminalia glaucescens Linn. & Combretaceae & & & $\mathrm{x}$ \\
\hline 68 & Terminalia macroptera Guill. \& Perr. & Combretaceae & $\mathrm{x}$ & & $\mathrm{x}$ \\
\hline 69 & Trichilia emetica Vahl subsp. suberosa J.J. & Meliaceae & & & $\mathrm{x}$ \\
\hline 70 & Uvaria chamae P. Beauv. & Annonaceae & & $\mathrm{x}$ & $\mathrm{X}$ \\
\hline 71 & Vernonia guineensis Benth. Var guineensis & Asteraceae & & & $\mathrm{x}$ \\
\hline 72 & Vitellaria paradoxa C. F. Gaertn. & Sapotaceae & & $\mathrm{x}$ & $\mathrm{x}$ \\
\hline 73 & Vitex doniana Sweet & Verbenaceae & $\mathrm{x}$ & $\mathrm{x}$ & $\mathrm{x}$ \\
\hline 74 & Waltheria indica Linn. & Sterculiaceae & & & $\mathrm{x}$ \\
\hline 75 & Ximenia americana Linn. & Olacaceae & & $\mathrm{x}$ & $\mathrm{x}$ \\
\hline 76 & Zanthoxylum Zanthoxyloides (Lam.) Zepern. \& Timler & Rutaceae & & & $\mathrm{x}$ \\
\hline
\end{tabular}




\section{DISCUSSION}

L'étude a permis de mettre en évidence 6 formations végétales que sont les forêts claires, les forêts galeries, les jachères, les savanes arborées/arbustives, les mares et les savanes herbeuses. Comme l'ont déjà mentionné Koulibaly (2008) et N'Dri (2011), ce sont les forêts claires qui constituent le type de forêt le plus répandu dans les régions de savane. Les espèces rencontrées corroborent celles de Hountondji (2008). Selon cet auteur, les forêts claires rassemblent un cortège relativement modeste d'espèces telles que : Detarium senegalense J.F.Gmel., Daniellia oliveri Hutch. et Dalz., Dialium guineense Willd., Afzelia africana Sm., Pterocarpus erinaceus Poir., Isoberlinia doka Craib ex Stapf et Khaya senegalensis (Desv.) A. Juss.. Les forêts galeries sont de dimensions plus modestes comme l'ont déjà constaté Ambé (2001). Les jachères occupent des superficies plus importantes. Les plus anciennes sont dominées par des arbustes, des arbres de Karité et des hautes herbes annuelles. Les plus jeunes jachères comportent encore les traces de cultures récentes dont le cotonnier et certaines cultures céréalières ainsi que quelques adventices telles que Hyptis suaveolens Poit. et Imperata cylindrica (Linn.) Raeuschel. En plus des espèces susmentionnées, quelques individus d'arbres de karité et de néré ont été épargnés par les populations sur les champs à cause de leurs fruits comestibles (Codjia et al., 2003).

L'analyse comparative avec l'ensemble de la flore de Côte d'Ivoire montre que le site est relativement peu diversifié. Il compte seulement $10,41 \%$ de la flore nationale. Les forêts claires enregistrent la plus grande richesse floristique. Ensuite, viennent les galeries forestières; les savanes herbeuses étant les plus pauvres. Ces résultats corroborent ceux de Sambou (2004) qui mentionnent que les savanes sont des écosystèmes relativement moins diversifiés. Dans ces écosystèmes, seules les espèces les mieux adaptées s'y développent et arrivent à se maintenir. En effet, la distribution spatiale des espèces est liée aux conditions écologiques et à des caractères qui leur sont propres (pouvoir de régénération, capacité d'adaptation aux contraintes du milieu). Elle résulte aussi de la pression exercée par d'autres facteurs comme la sécheresse, les feux de brousse, les défrichements, l'exploitation et le pâturage (Lykke et Sambou, 2002).

La présence d'espèces à statut particulier, confirme le rôle de conservation de la biodiversité que peut jouer cette région. Selon Adou Yao (2005), la présence des espèces à statut de conservation aide à justifier la mise en défens d'un site. Les efforts de conservation de ces espèces et des types de végétation associés doivent être prioritaires (Myers et al., 2000). Les écosystèmes identifiés sont aussi à l'origine d'un certain nombre de services rendus aux populations locales. Ces dernières associent aux plantes rencontrées des usages thérapeutiques, alimentaires et plusieurs autres usages (artisanat, construction...). En effet, les écosystèmes sont reconnus par plusieurs travaux de recherche pour l'abondance des biens qu'ils procurent : produits de subsistance et d'échange, aliments, médicaments, épices, résines, latex, bois de feu et, naturellement, bois d'œuvre avec tous ses dérivés (FAO, 2005; MEA, 2005). Parmi les services écosystémiques, l'approvisionnement en plantes médicinales constitue l'une des ressources précieuses pour la grande majorité des populations riveraines. Le fait que les régions du Nord constituent encore des réservoirs d'espèces médicinales a été déjà signalé par Dro et al. (2013). Ces résultats vont aussi dans le même sens que ceux de Jiofack et al. (2010) et Fokunang et al. (2011). Selon ces auteurs, ce sont plus de $80 \%$ de la population rurale qui s'en sert en Afrique pour assurer leurs soins de santé. Pour Ambé (2001) la connaissance et l'utilisation des plantes par les populations locales serait étroitement liée à trois facteurs principaux que sont la distribution des espèces concernées, la disponibilité dans le temps et le ou les usages 
que les populations en font. Les espèces à usage thérapeutique se rencontrent en savanes arborées et forêts claires alors que celles à usage alimentaire se rencontrent dans les jachères de la zone d'étude (Tengrela). Quant aux espèces à divers usages, les biotopes de fortes concentrations sont les forêts galeries. Des études complémentaires poussées doivent être menées pour mieux appréhender le rôle joué par les écosystèmes naturels de la zone soudanienne de la Côte d'Ivoire dans la conservation et l'utilisation durable de la biodiversité floristique et des plantes utilitaires en particulier.

\section{Conclusion}

L'étude réalisée sur la diversité et l'usage de la flore des formations végétales de Tengrela indique que la région est riche d'un important nombre d'espèces végétales à statut particulier dont la présence nécessite la création d'une zone refuge de biodiversité. Les usages des plantes rencontrées sur le site sont divers. Ce sont des ressources naturelles importantes en termes de services d'approvisionnement pour la population locale dont il faudra tenir compte dans les processus de création des réserves naturelles volontaires. Aussi, il serait important, lors des aménagements des écosystèmes dégradés, de faire des enrichissements avec des plantes à usage multiple, intégrant les exigences écologiques du milieu et les considérations économiques, culturelles et alimentaires des populations locales.

\section{CONFLIT D'INTERETS}

Les auteurs déclarent qu'il n'existe aucun conflit d'intérêts par rapport à cet article.

\section{CONTRIBUTIONS DES AUTEURS}

DO est le principal investigateur de ce travail et a participé à toutes les phases du travail ; DK et MST ont contribué à la collecte des données sur le terrain et à la rédaction de l'article ; YJCK a contribué aux analyses statistiques et à la correction de l'article ; KEN a contribué par sa lecture à l'amélioration et à la validation du manuscrit.

\section{REMERCIEMENTS}

Les auteurs de cet article voudraient traduire leur reconnaissance aux tradipraticiens et chefs de famille des villages de Papara et Doubasso du département de Tengrela qui ont bien voulu se soumettre aux questionnaires. Les remerciements vont également à l'endroit de Monsieur ASSI Yapo Jean, Technicien botaniste qui a contribué à l'identification des espèces végétales inventoriées. Les auteurs tiennent aussi à remercier les référés pour leurs remarques constructives qui ont permis l'amélioration du document.

\section{REFERENCES}

Adou Yao CY, N'Guessan EK. 2005. Diversité botanique dans le sud du parc national de Taï, Côte d'Ivoire. Afrique Science, 01(2) : 295-313.

Adou Yao CY. 2005. Pratiques paysannes et dynamique de la biodiversité dans la Forêt Classée de Monogaga (Côte d'Ivoire). Thèse de doctorat, Département Hommes Natures Sociétés, Muséum national d'histoire naturelle, Paris, France, 233 p.

Agbahungba G, Sokpon N, Gaoué OG. 2001. Situation des ressources génétiques forestières du Bénin. Atelier sous-régional FAO/IPGRI/ICRAF sur la conservation, la gestion, l'utilisation durable et la mise en valeur des ressources génétiques forestières de la zone sahélienne (Ouagadougou, 22-24 sept. 1998). Note thématique sur les ressources génétiques forestières. Document FGR F, 12p.

Aké-Assi L. 1988. Espèces rares et en voie d'extinction de la flore de la Côte d'Ivoire. Monogr. Syst. Bot. Missouri Botanical Garden, 25 : 461-463.

Aké-Assi L. 2001. Flore de Côte d'Ivoire 1, Catalogue Systématique, Biogéographie et Ecologie. Conservatoire et Jardin Botaniques : Genève, Suisse, 396 p. 
Aké-Assi L. 2002. Flore de Côte d'Ivoire 2, Catalogue Systématique, biogéographie et Ecologie. Conservatoire et Jardin Botaniques, Genève, Suisse ; 401p.

Ambé GA. 2001. Les fruits sauvages comestibles des savanes guinéennes de Côte-d'Ivoire : état de la connaissance par une population locale, les Malinkés. Biotechnologie, Agronomie, Société et Environnement, 5(1): 43-58.

Amon DEA, Soro D, Traore D. 2015. Evaluation de l'infestation des Loranthaceae sur les ligneux des agroécosystèmes de la région du SudComoé (Côte d'Ivoire). International Journal of Biological and Chemical Sciences, 9(4): 1822-1834. DOI : http://dx.doi.org/10.4314/ijbcs.v9i4.8

Barima YSS, Barbier N, Bamba I, Traore D, Lejoly J, Bogaert J. 2009. Dynamique paysagère en milieu de transition forêtsavane ivoirienne. Bois et forêts des Tropiques, 299: 15-25.

Chardon PA. 1981. Méthodes pratiques de dépouillement de questionnaires. Thèse de Doctorat ès Sciences économiques, Université de Neuchâtel, France, 136 p.

Codjia JTC, Assogbadjo AE, Ekué MRM. 2003. Diversité et valorisation au niveau local des ressources végétales forestières alimentaires du Bénin. Cahiers Agricultures, 12 : 1-12.

Condit R., Pitman N, Leigh EGJ, Chave J, Terborgh J, Foster RB. 2002. Betadiversity. In Tropical forest trees. Science, 295: 666 - 669. DOI: 10.1126/science. 1066854

Dro B, Soro D, Koné MW, Bakayoko A, Kamanzi K. 2013. Evaluation de l'abondance de plantes médicinales utilisées en médecine traditionnelle dans le Nord de la Côte d'Ivoire. Journal of Animal \& Plant Sciences, 17(3): 26312646.

DOI: http://www.m.elewa.org/JAPS.

Dugué P, Koné FR, Koné G. 2003. Gestion des ressources naturelles et évolution des systèmes de production agricole des savanes de Côte d'Ivoire: conséquences pour l'élaboration des politiques agricoles. Actes du colloque, 27-31 mai 2002, Garoua, Cameroun, 12p.

Escofier B, Pages J. 1989. Multiple factor analysis: results of three-year utilization. In Multiway Data Analysis. Elsevier Science Publishers B.V.: North-Holland; $277-285$.

FAO (Organisation des Nations Unies pour l'Alimentation et l'Agriculture). 2005. Situation des Forêts du Monde. FAO: Rome ; 115.

FAO. 2006. Global forest resource assessment 2005. Progresss towards sustainable forest management. Forestry Paper 147: 320 p.

Fokunang CN, Jiofack RB, Ngameni B, Asongalem E, Guedje NM, Kechia F, Tembe-Fokunang E, Ngoupayou J, Torimiro NJ, Gonsu KH, Sielinou V, Ndikum V, Tabi O, Ngadjui BT., Ngogang J, Asonganyi T, Abena OM, Nkongmeneck Lohoue J, Colizzi V, Angwafor III F. 2011. Traditional Medicine: Past, Present and Future Development Prospects and Integration in the National Health System of Cameroon. Accepted for publication by African Journal of Traditional, Alternative and Complementary Medecine, 8(3): 284-295.

Gafsi M. 2007. Exploitations Agricoles Familiales en Afrique de l'Ouest et du Centre: Enjeux, caractéristiques et éléments de gestion. Editions Quae. 125 p.

Guillaumet JL, Adjanohoun E. 1971. La végétation de la Cote d'Ivoire. In $L e$ Milieu Naturel de Côte d'Ivoire. Avenard J-MM, Eldin G, Girard J, Sircoulon P, Touchebeuf J-L, Guillaumet E, Adjanohoun A, Perraud Mémoires ORSTOM n $^{\circ} 50$ : Paris, France ; 161-263.

Hountondji YC. 2008. Dynamique environnementale en zones sahélienne et soudanienne de l'Afrique de l'Ouest : Analyse des modifications et évaluation de la dégradation du couvert végétal, Thèse de doctorat, faculté des sciences, département des sciences et gestion de 
l'Environnement, Université de Liège, 153 p.

Jiofack T, Fokunang C, Guedje N, Kemeuze $\mathrm{V}$, Fongnzossie E, Nkongmeneck BA, Mapongmetsem PM, Tsabang N. 2010. Ethnobotanical uses of medicinal plants of two ethnoecological regions Cameroon. International Journal of Medicine and Medical Sciences, 2(3): 60- 79. DOI: http://www.academicjournals.org/ ijmms

Kassi N, Aké-Assi E, Tiebre M. 2010. Biodiversité végétale et vitesse de la régénération de la forêt classée de Sanaimbo (Côte d'Ivoire). Sciences \& Nature, 7(2) : 195 - 206. DOI : http://dx.doi.org/10.4314/scinat.v7i2.5996 3

Koffi AA, Kouassi FA, N'Goran SBK, Soro D. 2014. Les Loranthaceae, parasites des arbres et arbustes : cas du département de Katiola, au nord de la Côte d'Ivoire. International Journal of Biological and Chemical Sciences, 8(6): 2552-2559. DOI : http://dx.doi.org/10.4314/ijbcs.v8i6.17

Koffi KJ, Kouassi AF, Yao CYA, Bakayoko A, Ipou IJ, Bogaert J. 2015. The present state of botanical investigations in Côte d'Ivoire. Biodiversity Informatics, 10(2): 56-64.

DOI: http://dx.doi.org/10.17161/bi.v10i2.5007

Konan KE. 2008. Conservation de la diversité végétale et activités humaines dans les aires protégées du sud forestier ivoirien : l'exemple du Parc National d'Azagny. Thèse Unique Université d'AbidjanCocody, Côte d'Ivoire, 269 p.

Koulibaly AV. 2008. Caractéristiques de la végétation et dynamique de la régénération, sous l'influence de l'utilisation des terres, dans des mosaïques forêts-savanes, des régions de la réserve de Lamto et du parc national de la Comoé, en côte d'ivoire. Doctorat de thèse unique de l'Université de Cocody-Abidjan, 216p

Lavergne S, 2004. Les espèces végétales rares ont-elles des caractéristiques écologiques et biologiques qui leur sont propres? Application à la conservation de la flore en
Languedoc-Roussillon, Acta Botanica Gallica, 151(3): 327-331. DOI : 10.1080/12538078.2004.10515434

Lykke AM, Sambou B. 2002. Dynamique des espèces ligneuses dans une savane soumise aux feux dans le parc National du Delta du Saloum, Sénégal. Am. Bot. Afr. Ouest, 02: 67 - 78

MEA (Millennium Ecosystem Assessment) 2005. Ecosystems and human well-being: desertification synthesis. World Resources Institute. Washington, DC: Island press.

MINEEF (Ministère de l'environnement et des eaux et forêts). 2007. Diversité biologique de la Côte d'Ivoire. Ministère de l'Environnement et des Eaux et Forêts ; 10-20.

Myers N, Mittermeier RA, Mittermeier CG, Da Fonseca GAB, Kent J. 2000. Biodiversity hotspots for conservation priorities. Nature, 403: 853-858. DOI : 10.1038/35002501

N'Da D, Adou Yao CY, N'Guessan KE, Kone M, Sagne YC. 2008. Analyse de la diversité floristique du parc national de la Marahoué, Centre-Ouest de la Côte d'Ivoire. Afrique Science, 4(3): 552-579.

N'Guessan KE. 2010. Etudes d'impact environnemental et social du projet d'expansion de la mine de Bonikro sur les gisements satellites de Hiré Rapport d'étude floristique, CNF, $61 \mathrm{p}$.

N'Guessan KE. 2012. Etudes d'impact environnemental et social du projet de construction de la ligne $90 \mathrm{kV}$ d'alimentation du site minier d'Agbaou. Rapport d'études floristiques, CNF, 56 p.

N'Guessan KE. 2013. Etudes d'impact environnemental et social du projet de construction de la clôture du Parc National du Banco, Abidjan. Rapport d'études floristiques, $\mathrm{CNF}, 47 \mathrm{p}$.

N'DRI AB. 2011. Interaction termite-feu et dynamique de la végétation en savane (Lamto, Côte d'Ivoire). Thèse de doctorat. Paris $6.315 \mathrm{p}$.

Perraud A. 1971. Les sols. In Le milieu naturel de Côte d'Ivoire. Avenard J-MM, 
Eldin G, Girard J, Sircoulon P, Touchebeuf J-L, Guillaumet E, Adjanohoun A, Perraud Mémoires ORSTOM n ${ }^{\circ} 50$ : Paris, France ; 269-390.

Sambou B. 2004. Evaluation de l'état, de la dynamique et des tendances évolutives de la flore et de la végétation ligneuses dans les domaines soudaniens et sub-guinéen au Sénégal, Mémoire de Doctorat, Université Cheikh Anta Diop, Faculté des sciences et techniques, institut des sciences de l'environnement, $230 \mathrm{p}$.

Schmidt R. 1990. The management of tropical forest for substainable development. Asenan Seminar - Jakarta, 24-25 January 1990, 21p.

Teyssèdre A. 2004. Vers une sixième grande crise d'extinctions ? Biodiversité et changements globaux : enjeux de société et défis pour la recherche. Ministère des Affaires Etrangères-ADPF, Paris, 36p.

Troupin G. 1966. Étude phytosociologique du Parc National de l'Akagera et du Rwanda oriental. Recherche d'une méthode d'analyse appropriée à la végétation d'Afrique intertropicale. Liège (Belgique), $293 \mathrm{p}$.

UICN (Union Internationale pour la Conservation de la Nature). 2015. Red List of Threatened Species. <www.iucnredlist.org>. Downloaded on 13 August 2015.

Vroh BTA. 2013. Evaluation de la dynamique de la végétation dans les zones agricoles d'Azaguié (Sud-est Côte d'Ivoire). Université Félix-Houphouët-Boigny, UFR Biosciences, Thèse Unique de Botanique, Côte d'Ivoire, 131p.

Vroh BTA., Tiébré MS, Ouattara D, N'Guessan KE. 2014. La réserve forestière Dékpa d'Agbaou, un exemple de conservation de la diversité végétale sur les sites miniers de la Côte d'Ivoire, International Journal of Innovation and Applied Studies, 6(2): 162. DOI : http://www.ijias.issr-journals.org 